\title{
Discharge Patterns of Single Reticular Neuron Synchronized with Electrocortical Spike Induced by Pentetrazol
}

\author{
Takeo Fukuda, Nobuo Katsuda and Kiyoshi Tanaka \\ Department of Pharmacology (Prof. K. Tanaka), \\ Faculty of Medicine, Kyushu University, Fukuoka
}

\begin{abstract}
The majority of the midbrain reticular neurons of the rat altered their discharge patterns synchronously with a cortical EEG spike induced by intravenous injection of pentetrazol. The patterns were classified into four types, i.e., facilitated, inhibited, mixed and unaffected types.

Intravenous lidocaine suppressed both facilitatory and inhibitory components in the firing pattern. In particular, it reversed inhibitory to facilitatory pattern in some neurons. This may support the hypothesis that local anestheties are liable to depress some inhibitory process in the central nervous system.
\end{abstract}

It is considered that the neurons of reticular formation of the brain stem play an important role in the convulsion and the mechanism of action of anticonvulsant drugs (Kreindler et al.; Tanaka and Ito $^{2}$ ).

On the other hand, the relationship between cortical unit activities and an electrocortical spike or seizure discharges has been studied by several investigators (Weir and Sie, ${ }^{3}$ Feher et al., ${ }^{4}$ and Fukuda et $a l .{ }^{5}$ ). The present experiments aim to find out some correlation between reticular unit activity and an electrocortical spike and to test how drugs modify it, since this question remains unsettled.

\section{Methods}

Unanesthetized 24 adult rats of both sexes, immobilized with decamethonium, were employed as experimental animals. Firing of a single neuron was picked up with an extracellular tungsten microelectrode from the midbrain reticular formation at the level of the red nucleus, $1.5 \mathrm{~mm}$ lateral to the midline. Electroencephalogram (EEG) was led from the motor cortex with a bipolar electrode. Both unitary discharge and cortical EEG were taped continuously under monitoring with a double-beam oscillograph and recorded photographically with a kymograph camera if necessary.

Pentetrazol (Metrazol) was slowly injected intravenously in a dose of $20-30 \mathrm{mg} / \mathrm{kg}$, which was sufficient to elicit a sporadic single cortical spike without inducing a hypersynchronized seizure discharge.

To test the effect of a drug on the reticular unitary activity synchronized with a cortical spike induced by pentetrazol, firing patterns of the same neuron were compared before and after intravenous administration of the testing drugs such as lidocaine, diphenylhydantoin, etc. At least one hour interval was always kept between the first and the second provocation of cortical spikes by pentetrazol.

Received for publication, September 2, 1969 . 


\section{Results}

1. Firing patterns of reticular neurons associated with a cortical EEG spike

Firing patterns of reticular neurons associated with full size of sporadic cortical spikes induced by pentetrazol were noted to be almost fixed on the same neuron. They were classified into four types as follows and schematically illustrated in Fig. 1.

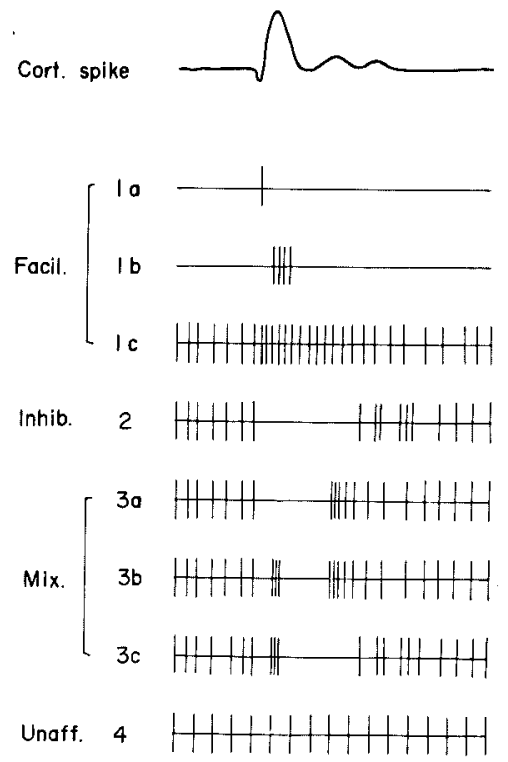

Fig. 1. Schematic illustration of the patterns of reticular neuron discharge synchronized with pentetrazol-induced cortical EEG spike.

(1) In the first type, the discharge frequency increased more or less clearly synchronizing with the generation of a cortical spike (facilitated type). This type was further divided into three subgroups: a) A single discharge in a silent neuron (1a type), b) a short burst of firing in a silent neuron (1b type), and c) increase in the firing rate in a spontaneously discharging neuron (lc type).

(2) In the second type, the firing rate decreased simply synchronized with the generation of a cortical spike (inhibited type). Occasionally, a complete cease of discharge was noted.

(3) The third type was the combined pattern of facilitation with inhibition (mixed type). This was divided into three subgroups: a) Inhibition followed by a rebound after-facilitation ( 3 a type), b) inhibition interpolated by a short burst of firing and followed by a rebound after-facilitation ( $3 b$ type), and c) inhibition interpolated by a short burst of firing without after-facilitation (3c type). 
(4) The fourth type represents the neurons which discharged almost indifferently to a cortical spike (unaffected type). Usually such a neuron showed a quite regular spontaneous firing.

The number of neurons involved in each type is shown in Table 1, indicating the largest in mixed type $(39 \%)$ and the least in unaffected type $(13 \%)$. The depth distribution range of each type of neurons estimated from a micromanipulator reading beneath the cortical surface is also listed in the table, showing that the neurons of inhibited type were predominantly distributed in the ventral region of the reticular formation, whereas the neurons of facilitated type were rather in dorsal region.

TABLE 1. Classification of the patterns of reticular neuron discharges

\begin{tabular}{l|c|c|c}
\hline \multicolumn{1}{c|}{ Type } & Subgroup & Number of unit & Depth $(\mu)$ \\
\hline \multirow{3}{*}{ 1. Facilitated } & la & 2 & $430-450$ \\
& Ib & 3 & $480-550$ \\
Ic & 4 & $530-590$ \\
\hline 2. Inhibited & & 10 & $530-680$ \\
\hline & $3 \mathrm{a}$ & 4 & $470-530$ \\
3. Mixed & $3 \mathrm{~b}$ & 4 & $490-580$ \\
& $3 \mathrm{c}$ & 7 & $550-610$ \\
\hline 4. Unaffected & & 5 & $450-550$
\end{tabular}

\section{Influences of lidocaine and other drugs on the unitary firing patterns}

Effects of intravenous lidocaine on various discharge patterns above-mentioned were tested. In several experiments, diphenylhydantoin and hexobarbital were also applied.

In a neuron of la type, $8 \mathrm{mg} / \mathrm{kg}$ of lidocaine eliminated a single discharge synchronized with a cortical spike. This effect vanished after 10 minutes (Fig. 2). Similar suppressive effect of this drug on the facilitatory discharge was recognized

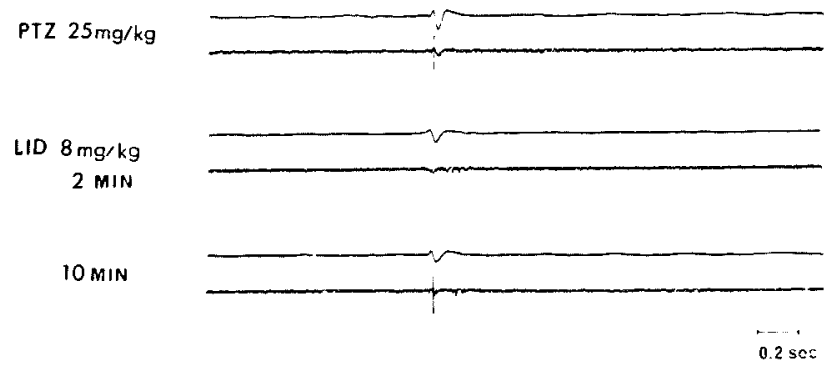

Fig. 2. Depressant effect of lidocaine on a la type neuron.

Upper tracing: cortical EEG. Lower tracing: reticular unit firing. PTZ: pentetrazol. LID: lidocaine. 
on all other six facilitated neurons (type 1) tested, including two la, three $1 \mathrm{~b}$ and one 1c types. The effect of hexobarbital and diphenylhydantoin on facilitated neurons was also analogous to that of lidocaine.

In two neurons of inhibited type (type 2), lidocaine caused no change in the firing pattern in one, but reversed the inhibitory pattern to facilitatory (type $1 b$ ) on the other. No such a reversal was observed, however, under the effects of hexobarbital or diphenylhydantoin.

In all the seven neurons of mixed type (type 3), lidocaine effectively suppressed or completely eliminated the facilitatory components such as a short burst of firing during a cortical spike or a rebound after-facilitation. In two neurons of them, on the other hand, it reversed the inhibitory phase immediately succeeding to the short burst firing to facilitatory one.

Fig. 3 reveals an example of the above-mentioned effect of lidocaine on a mixed type of neuron (type $3 b$ ), in which the short burst of firing and rebound after-facilitation were abolished, and the inhibitory phase between them was reversed to facilitation. In this case the frequency of spontaneous discharge was diminished after lidocaine and recovered within 30 minutes. Similar effect was observed in many other neurons, though it was usually preceeded by a transient and remarkable increase in spontaneous discharge rate which lasted about 30 seconds immediately after the injection.

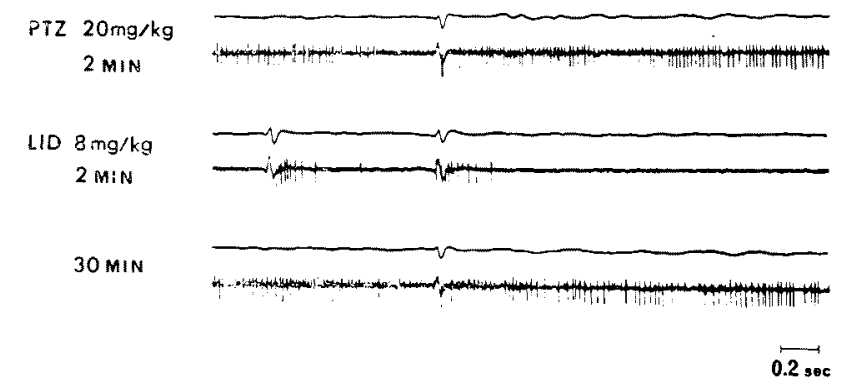

Fig. 3. Peversal of discharge pattern by lidocaine in a $3 b$ type neuron.

Diphenylhydantoin behaved somewhat differently from lidocaine on the same neuron. While it suppressed both spontaneous discharges and a short burst of firing during a cortical spike just as lidocaine did, it did not reverse the successive short inhibition to facilitation. In other words, it shifted the pattern of $3 \mathrm{~b}$ type to 3a (Fig. 4). Besides, a late and prolonged jnhibition became evident under the effect of this drug, which was not disclosed under the effect of lidocaine. In other two neurons of type 3 , the suppression of the facilitatory components and disclosure of the late and prolonged inhibition were found to be the general prevailing features of the effect. In short, diphenylhydantoin was thought io be effective in suppressing facilitatory components and in producing the inhibitory predominance in the firing pattern. 


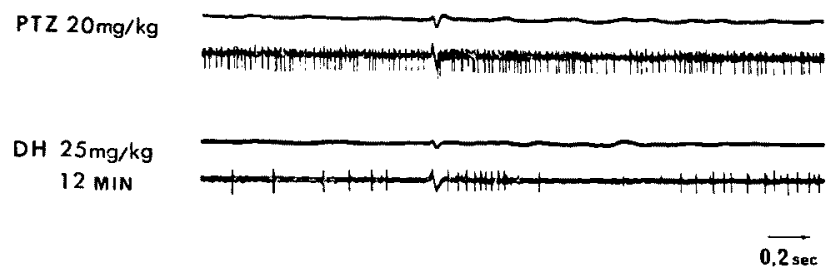

Fig. 4. Effect of diphenylhydantoin(DH) on the same neuron in Fig. 3.

In neurons of fourth type, the pattern was affected neither by lidocaine nor by hexobarbital, although the spontaneous discharge rate was somewhat reduced (Fig. 5).

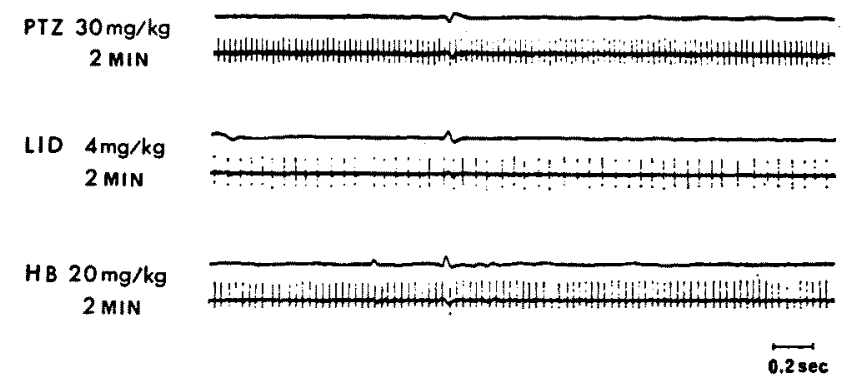

Fig. 5. Effect of lidocaine and hexobarbital $(\mathrm{HB})$ on a 4 th type neuron.

\section{Discussion}

The present experiment demonstrated that the majority of reticular neurons except for only 13 per cent of unaffected type were involved in the neuronal activity synchronized with the generation of the cortical EEG spikes induced by pentetrazol. Furthermore, these activated neurons were distributed diffusely in the whole depth of the reticular formation. This would strongly indicate some of the important roles of the reticular formation in convulsive activity and in anticonvulsant mechanisms of drugs as already pointed out by others (Kreindler et al., ${ }^{1}$ Tanaka and Ito $^{2}$ ).

Notwithstanding that a relatively rigid coupling has been found between cortical EEG spikes and many cortical unit activities (Weir and Sie, ${ }^{3}$ Feher et al., ${ }^{4}$ Fukuda et $a .^{5}$ ), the present experiments disclosed a rather dispersed relationship between cortical spikes and reticular unit activities. The dispersion of the firing pattern of reticular neurons seems to be a manifestation of the convergence of various amounts of facilitatory and inhibitory inputs on a single neuron. Furthermore, analysis in the pattern distribution in the reticular formation indicated a greater participation of the inhibitory components at the ventral region than at the dorsal area, though its physiological significance is not clear. 
As to the action of local anesthetics on the central nervous system, an assumption that they might preferentially depress an inhibitory process was presented by Tanaka and Yamasaki. ${ }^{6}$ They postulated the hypothesis for cortical neurons, and it was adopted by De Jong et al. ${ }^{7}$ for spinal neurons. The appearance of spontaneous discharges in a naturally silent reticular neuron following lidocaine could be interpreted also by the release from some neuronal inhibitory process as pointed out already (Fukuda and Tanaka ${ }^{8}$ ).

The present experiment demonstrated an example in which lidocaine suppressed the inhibitory component and converted it to facilitation in the firing pattern of a reticular neuron associated with a cortical EEG spike induced by pentetrazol. This may serve as another evidence of the above-mentioned dis-inhibitory mechanism, and may indicate that the assumption would be warranted for all central nervous structures.

It could not still be overlooked that lidocaine also suppressed some facilitatory components in the firing pattern such as a short burst discharge during a cortical spike or a rebound after-facilitation. Similar phenomenon was observed on reticular neurons responding to subthalamic stimulation (Fukuda and Tanaka ${ }^{8}$ ).

Considering the dual effect of lidocaine above-mentioned, it seems to be reasonable to postulate that the suppression of both facilitatory and inhibitory components of various spacio-temporal features which interacted on a single neuron could easily result in a reversal of firing pattern depending on the different vulnerability of each component to lidocaine. In this regard, the former finding of the facilitatory response of reticular neurons which was quite resistant to lidocaine (Fukuda and Tanaka ${ }^{8}$ ) would strongly support the possibility of frequent manifestation of the neuronal dis-inhibitory phenomena by this drug.

In contrast, the effect of diphenylhydantoin is conceivable to suppress mainly some facilitatory components, resulting in a predominance of inhibitory one, though further accumulation of data is necessary for a more detailed analysis.

\section{References}

1) Kreindler, A., Zuckermann, R., Steriade, M. \& Chimion, D. Electroclinical features of convulsions induced by stimulation of brain stem. $J$. Neurophysiol., 1958, 21, 430-436.

2) Tanaka, K. \& Ito, I. Inhibitory effects of anticonvulsants on the ascending reticular activating system. Yonago Acta med., 1958, 3, 112-114.

3) Weir, B. \& Sie, P.G. Extracellular unit activity in cat cortex during the spike-wave complex. Epilepsia, 1966, 7, 30-43.

4) Feher, O., Halasz, P. \& Mechler, F. The mechanism of origin of cortical convulsive potentials. EEG clin. Neurophysiol., 1965, 19, 541-548.

5) Fukuda, T., Manmaru, S., Kazama, O., Oka, I. \& Ebata, K. Relationship between components of cortical seizure wave and unit activities, Psych. Neur. Jap. (Jap.), 1968, $70,747$.

6) Tanaka, K. \& Yamasaki, M. Blocking of cortical inhibitory synapses by intravenous lidocaine. Nature, 1966, 209, 207-208.

7) De Jong, R.H., Robles, R. \& Carbin, R.W. Central actions of lidocaine--synaptic transmission. Anesthesiology, 1969, 30, 19-23. 
8) Fukuda, T. \& Tanaka, K. The effect of subthalamic stimulation on the activity of reticular neurons in rats. Fukuoka Acta med., 1968, 59, 401-404. 